\title{
Hepatitis B Sero-Conversion Rates among HIV Positive Children Immunized with Pentavalent Vaccine in Bamenda Health District, Cameroon
}

\section{Lem Edith Abongwa ${ }^{1 *}$, Forwang Lisette Nkengbeza ${ }^{1}$, Clauvis Yengo ${ }^{2}$, Signang Alberic ${ }^{3}$ and Mabeyonga Berenice Fokong ${ }^{1}$}

${ }^{1}$ Department of Biological Sciences, Faculty of Science, University of Bamenda, $N$. W. Region, Cameroon

${ }^{2}$ Department of Biochemistry, University of Yaounde I, Cameroon

${ }^{3}$ Mbingo Baptist Hospital, Cameroon Baptist Convention Health Services, BMP 41 via Bamenda, Cameroon

*Corresponding Author: Lem Edith Abongwa, Department of Biological Sciences, Faculty of Science, University of Bamenda, N. W. Region, Cameroon.
Received: June 04, 2021;

Published: July 06, 2021

(C) All rights are reserved by Lem Edith

Abongwa., et al.

\begin{abstract}
Background/Aims: Immunosuppression caused by the human immunodeficiency virus (HIV) alters the pathogenesis of the hepatitis B virus (HBV) infection thereby increasing HBV prevalence. Globally, HIV and HBV remain major public health concerns. Immunization is an effective method of preventing childhood infection. This cross-sectional study aimed at assessing the effectiveness of the pentavalent vaccine in the prevention of HBV transmission in HIV-positive children.

Methods: Children who gave their assent with the consent of their parents/guardians were recruited into the study. Sociodemographic information was collected using a close-ended questionnaire. Serum samples were used to test for serological markers of HBV. One hundred and fifty-six children were enrolled between March and July 2020. Data analyses were done using SPSS version 23.0 .

Results: The age range of the participants were 0.8 months to 15 years with a mean \pm standard deviation of $9.6 \pm 3.8$ years. The prevalence of HBV among the study participants was $2(1.3 \%)$, and it was insignificantly $(\mathrm{p}=0.25)$ higher in females $(2.1 \%$ vs $0.0 \%)$ compared to their male counterparts. As concerns age group, <6years recorded the lowest prevalence $0(0.0 \%)$ and this was insignificant $(P=0.88)$. HBV prevalence was highly significant $(p=0.001)$ among children whose parents/guardians were positive $2(15.4 \%)$ for HBV

Conclusions: The use of the pentavalent vaccine is effective in preventing HBV and all babies born to HBV-positive pregnant women should be followed up to ensure that they receive the monovalent vaccine at birth and 4 weeks after birth alongside all the required vaccines.
\end{abstract}

Keywords: Children; HBV; HIV; Pentavalent Vaccine

\section{Abbreviations}

EPI: Expanded Program of Immunization; HAART: Highly Active Antiretroviral Therapy; HBsAg: Hepatitis B Surface Antigens; HBV:
Hepatitis B Virus; HIV: Human Immunodeficiency Virus; SSA: SubSaharan Africa 


\section{Introduction}

HIV alters the pathogenesis of viral hepatitis infections, thereby increasing the prevalence of Hepatitis B in HIV-infected persons. These blood-borne viruses share common routes of transmission which include: sexual contact, direct contact with infected blood and bodily fluids, and through mother to child transmission. In the pre- highly active antiretroviral therapy (HAART) era, several studies showed that HIV/HBV co-infection might increase the likelihood of more severe outcomes of HBV infection [1-3]. HBV/HIV prevalence remains high in several African countries (5 - 40\%), with a range of $6-17.5 \%$ co-infection among pregnant women in Cameroon $[4,5]$. As such this high prevalence requires the monitoring of HBV among children living with HIV to provide adequate pediatric care.

HBV infection is a major cause of morbidity and mortality in sub-Saharan Africa (SSA), despite the availability for the last three decades of a safe and effective vaccine [6]. Infection with HBV is a public health problem worldwide, with more than 350 million chronic carriers with $88 \%$ of them residing in SSA and Cameroon being considered as one of the most affected countries [4,6,7]. Hepatitis $B$ is a common serious liver infection that can lead to liver failure, cirrhosis (scarring), or liver cancer with a mortality rate of about $25-30 \%$ as a result of the consequences of chronic infection $[2,8]$. Although HBV is 40 - 100 times more infectious than HIV, public and professional awareness of hepatitis B remains low compared to HIV and other infections [3,9].

In Cameroon, the prevalence of hepatitis B surface antigens (HBsAg) positivity varies from $7.7 \%-17.5 \%$ among pregnant women with a relatively moderate pediatric HBV prevalence [9]. This moderate pediatric HBV prevalence is probably due to the wide pediatric coverage of anti-HBV vaccination $[5,10]$. Vertical transmission of HBV is mucosal and caused by perinatal exposure to infected maternal blood and body fluids at the time of delivery. However, transplacental transmission and transmission via breastfeeding are rare $[2,4]$. As such infants born to HBV-infected mothers are at high risk of HBV transmission and thus are expected to be given hepatitis B vaccine within 24 hours of birth that will provide marginal additional protection beyond $[3,4]$. Despite the advantages of the vaccine, routine screening in antenatal clinics in Cameroon does not include the hepatitis B surface antigen (HBsAg) screening compared to the developed world [8].
Interventions to prevent HBV vertical transmission are highly cost-effective since they reduce both short-term adverse outcomes and long-term morbidity and mortality $[2,11]$.

Vaccination is the most simple and cost-effective preventive intervention in the prevention of infectious diseases, especially in children [9,12]. The Expanded Program of Immunization (EPI) started in Cameroon in 1976 and in response to WHO recommendations, Cameroon introduced the hepatitis B vaccine into their EPIs in 2005 [8]. As such the EPI can protect children against hepatitis B, tuberculosis, diphtheria, tetanus, poliomyelitis, pertussis, Type b Hemophilus influenza infections, pneumococcal infections, diarrhea caused by rotavirus, measles, yellow fever, and rubeola $[8,12]$.

Studies elsewhere have highlighted a high protection rate of about $92 \%$ among children immunized with three injections of the anti-HBV vaccine (given at 6,10 and 14 weeks of age) to provide a lifetime of protection $[2,8,13]$. Given the importance of Hepatitis B vaccination in reducing the $\mathrm{HBV}$ morbidity and mortality rates in children [11] this study aimed at evaluating the effectiveness of the pentavalent combination vaccine in preventing HBV transmission among HIV positive children.

\section{Materials and Methods}

Study design: This study was a cross-sectional analytical study conducted over a period of 4 months from March to July 2020 in the pediatric HIV Unit of the Bamenda Regional Hospital. Pre-tested questionnaires were filled by all parents or guardians of children/ infants after obtaining consent. Socio-demographic and clinical information collected on the infants included age, sex, school attendance, level of education, and vaccination status. Children who were positive for HBV were referred for further assessment and management. The sample size $(\mathrm{N})$ was determined to be 37 using the formula: $\mathrm{z}^{2} \mathrm{p}(\mathrm{p}-1) / \mathrm{d}^{2}$ where $\mathrm{z}$ is the significance threshold; 1.96 for a $95 \%$ confidence level, $d$ is the error margin; $5 \%$, and p; $2.41 \%$, is the prevalence of HBV in children in Cameroon [5]. Only children who had completed their childhood vaccination were recruited in the study.

Ethical considerations: Administrative authorization and ethical clearance were obtained from the Bamenda Regional hospital and Regional hospital's ethical review board respectively. An informed 
consent form was obtained from either parents or guardians.

HBV testing: A total of $5 \mathrm{~mL}$ of whole blood was collected in dry tubes by venipuncture from each participant and centrifuged to obtain serum. HBV serological testing targeting HBsAg was performed as per the manufacturer's instructions using Diaspot HBsAg test Kit-Rapid Labs, United Kingdom. Results were reported either as positive, negative, or invalid.

\section{Data analysis}

Data analysis was done using SPSS version 23.0 (SPSS Inc. Chicago, IL, USA). Results are presented as count (percent) in tables and bar charts. Chi-square was used to look for an association between HbsAg and other categorical variables. The level of significance in hypothesis testing was set at $5 \%$.

\section{Results and Discussion}

A total of 161 children were recruited and 156 (96.9\%) provided complete information. The age range of the participants was 0.8 months to 15 years with a mean \pm standard deviation of $9.6 \pm 3.8$ years. Most of the participants belonged to the age group $>10$ years $70(44.9 \%)$, and were female 94 (60.3\%), had primary level of education 85 (54.5\%)and were living with their parents 102 (65.4\%). More than $50 \%$ of parents or guardians were HBV negative 143 $(91.7 \%)$ as shown in table 1.

\begin{tabular}{|l|c|c|c|}
\hline Characteristics & Variable & Frequency & Percent \\
\hline \multirow{4}{*}{ Age group in years } & $<6$ & 17 & 10.9 \\
\cline { 2 - 4 } & $6-10$ & 69 & 44.2 \\
\cline { 2 - 4 } & $>10$ & 70 & 44.9 \\
\hline \multirow{4}{*}{ sex } & Female & 94 & 60.3 \\
\cline { 2 - 4 } & Male & 62 & 39.7 \\
\hline \multirow{4}{*}{ Level of education } & None & 13 & 8.3 \\
\cline { 2 - 4 } & Nursery & 4 & 2.6 \\
\cline { 2 - 4 } & Primary & 85 & 54.5 \\
\cline { 2 - 4 } & Secondary & 54 & 34.6 \\
\hline \multirow{3}{*}{ Caregiver } & Guardian & 54 & 34.6 \\
\cline { 2 - 4 } & Parent & 102 & 65.4 \\
\hline \multirow{2}{*}{$\begin{array}{l}\text { HBV status of parent/ } \\
\text { guardian }\end{array}$} & Negative & 143 & 91.7 \\
\cline { 2 - 4 } & Positive & 13 & 8.3 \\
\hline
\end{tabular}

Table 1: Baseline characteristics of the study population $(\mathrm{N}=156)$.
The prevalence of HBV among the study participants was 2 $(1.3 \%)$ and it was insignificantly $(\mathrm{p}=0.24)$ higher in females 2 (2.1\%) than the male counterpart (Figure 1$)$. As concerns the age group, this prevalence was lowest $0(0 \%)$ in the age group $<6$ years when compared to the $>6$ years age group (Figure 2 ), similarly, this difference was not significant $(\mathrm{P}=0.83)$.

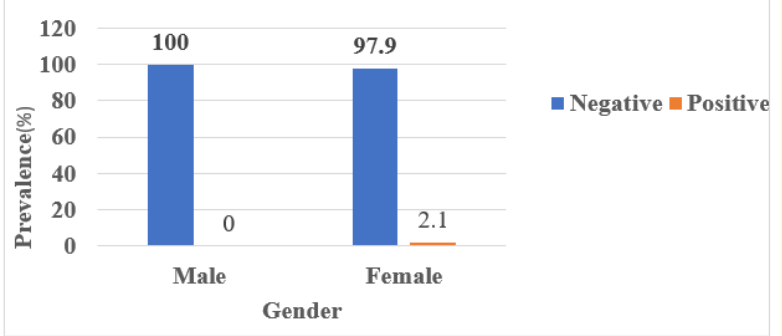

Figure 1: Prevalence of HBV by gender. $\chi 2=1.34 \mathrm{p}=0.25$.

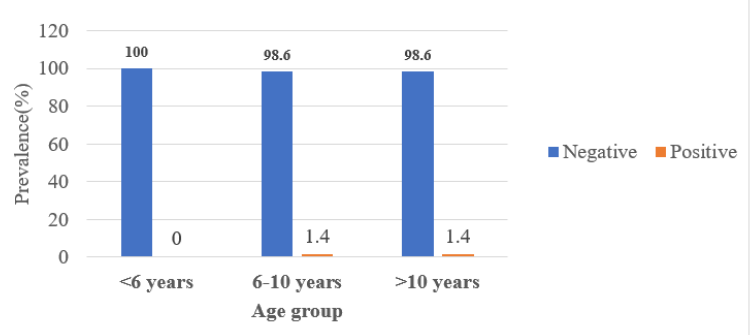

Figure 2: HBV prevalence by age group. $\chi 2=0.25 \mathrm{p}=0.88$.

HBV was highly significant $(\mathrm{p}=0.001)$ among children whose parents were positive for HBV 2 (15.4\%) than those with HBV negative parents/guardians $0(0 \%)$ as shown in table 2 .

We recorded a low prevalence of $1.3 \%$ in this study. The low rate of HBsAg carriers among children who received the complete dose of HBV vaccine suggests a positive impact of $H B V$ vaccination. This has been shown to reduce infant morbidity and mortality. Similar to other studies Hepatitis B vaccination is the most effective preventive intervention against infection with HBV and its complications 


\begin{tabular}{|l|c|c|c|c|}
\hline \multirow{2}{*}{$\begin{array}{c}\text { HBV status } \\
\text { of Parent/or } \\
\text { guardian }\end{array}$} & $\begin{array}{c}\text { ChBV } \\
\text { Negative } \\
\text { test (\%) }\end{array}$ & $\begin{array}{c}\text { HBV } \\
\text { Positive } \\
\text { (\%) }\end{array}$ & $\chi^{2}$ & p-value \\
\hline Negative & $143(100)$ & $0(0)$ & \multirow{2}{*}{22.29} & 0.0001 \\
\hline Positive & $11(84.6)$ & $2(15.4)$ & & \\
\hline
\end{tabular}

Table 2: Assessing the prevalence of HBV in children concerning parent/caregiver HBV status.

$[3,6,9,11,13]$. Before the introduction of the EPI program, previous studies in Cameroon showed an overall HBsAg prevalence of about $19.9 \%$ in children [14]. The low prevalence of hepatitis B surface antigen among children suggests a positive impact of HBV vaccinations similar to the $0.7 \%$ prevalence recorded in a study carried out in another town in Cameroon [8]. Additionally, results from other studies recorded a decrease in the prevalence of $\mathrm{HBV}$ from a range of 19.8 - 44\% prior to HBV vaccination to that of 1.3 - 9.8\% [13-15]. Furthermore, the low prevalence of HBV in HIV-positive children could also be attributed partly to maternal ART that contains tenofovir and lamivudine which are regimens used in treating chronic HBV infection [5].

Although studies have shown that HBV vaccination is efficient up to the age of 10 years and above [16,17], in this study the high prevalence seen in the age group $>6$ years indicates that seroprotection rates decrease with increasing age as reported by $[18,19]$. This is in line with previous findings from a similar study carried in Cameroon and elsewhere which state that poor immunological response to HBV-vaccine is possible with HIV-infected children thus signifying risks of HBV-infection despite complete vaccination $[5,20]$. Therefore, there is a likelihood that HBV infection can occur primarily by horizontal transmission between siblings and playmates rather than vertically from mother to child [6]. Therefore, it is of prime importance that the administration of HB vaccine challenge dose at 1 year be given after the principal EPI schedule doses (at 6, 10, and 14 weeks of age) to all children as recommended by WHO [21]. This challenge dose vaccine will induce strong anamnestic responses which are well tolerated $[9,16,18,22]$ and thus will prevent horizontal HBV transmission during childhood and not during maternal-infant transmission at birth [6]. Additionally, complete protection in all the participants was not registered in this study because studies have shown that response to anti-HBV vaccine varies according to HIV status, thereby indicating a high vulnerability to HBV among children living with HIV as described in a similar study in Cameroon [5].

The high prevalence recorded in children whose parents were also positive for HBV indicates that vertical transmission is also a key factor driving endemic HBV infection. Similar results have also been recorded in other SSA with a maternal HBV seroprevalence rate of $>8 \%[2-4,13]$. However, in Cameroon HBV test is not a routine test in antenatal care (ANC). In addition, as compared to all routine tests that are done free of charge, due to the cost of the HBV test most women are not willing to pay for the test. Moreover, the high cost of HBV treatment also has a negative effect on the prevention of MTCT since less than 1\% of pregnant women will be treated for HBV infection during pregnancy [2]. Therefore, it will be necessary to include the HBV test as a requirement of the ANC package and its treatment highly subsides for pregnant women.

As such the administration of monovalent vaccine at birth and 4 weeks after birth in addition to the continuation with pentavalent vaccine as part of the routine schedule in children born to HBV positive mothers may overcome some of the obstacles in preventing vertical or through horizontal HBV transmission (exposure to infected blood), especially from an infected child to an uninfected child during the first five years thus everybody needs to be vaccinated $[2,3,13]$.

\section{Conclusions}

Active immunization is the cornerstone of preventing HBV transmission. Integrating HBV services with antenatal care is required to minimize the vertical transmission of HBV. Screening all pregnant women for HBV infection, starting antiviral therapy for those who meet treatment criteria, introducing the HBV birth dose vaccine and increasing overall coverage of vaccine are all required to minimize the transmission of HBV.

\section{Recommendations}

Free and compulsory antenatal testing for HBV should be included in the ANC package in Cameroon. The administration of the birth dose HBV vaccine to all children born of HBV-positive mothers should be made mandatory. 


\section{Limitation of the Study}

Lack of funds to carry out HBV genotyping and measuring the level of immunological memory response was not done. As such we cannot for inevitability says that the HBV vaccination is still efficient after six years.

\section{Acknowledgments}

The authors are grateful to the hospital administration, staff of the paediatric HIV unit, the participants as well as the parents or guardians of the participants.

\section{Conflict of Interest}

No conflict of interest to declare.

\section{Bibliography}

1. Okwen Mbah P., et al. "Hepatitis B vaccination for reducing morbidity and mortality in persons with HIV infection". Cochrane Database of Systematic Reviews 10 (2014).

2. Dionne-Odom Jodie., et al. "Predictors of infant hepatitis b immunization in Cameroon: data to inform the implementation of a hepatitis b birth dose". The Pediatric Infectious Disease Journal 37.1 (2018): 103.

3. Mandal Sema. "Introduction of universal infant hepatitis B immunisation in the UK-paving the way to elimination". Human Vaccines and Immunotherapeutics 15.2 (2019): 440-443.

4. Abongwa Lem Edith and Penn Kenneth. "Assessing prevalence and risk factors of hepatitis B surface antigen amongst pregnant women attending antenatal clinic in the Northwest Region of Cameroon". European Journal of Research in Medical Sciences 4.1 (2016).

5. Joseph Fokam., et al. "Hepatitis B infection and risk factors among children living with HIV in Yaounde, Cameroon: an integrated management". BMC Pediatrics 19.1 (2019): 1-8.

6. Andersson MI., et al. "The epidemiology of hepatitis B virus infection in HIV-infected and HIV-uninfected pregnant women in the Western Cape, South Africa". Vaccine 31.47 (2013): 5579-5584.

7. Kowo Mathurin Pierre., et al. "Prevalence of hepatitis $C$ virus and other blood-borne viruses in Pygmies and neighbouring
Bantus in southern Cameroon". Transactions of the Royal Society of Tropical Medicine and Hygiene 89.5 (1995): 484-486.

8. Bekondi Claudine., et al. "HBV immunization and vaccine coverage among hospitalized children in Cameroon, Central African Republic and Senegal: a cross-sectional study". BMC Infectious Diseases 15.1 (2015): 1-9.

9. Faingezicht Idis., et al. "Primary and booster vaccination with DTPw-HB/Hib pentavalent vaccine in Costa Rican children who had received a birth dose of hepatitis B vaccine". Revista Panamericana de Salud Pública 12 (2002): 247-257.

10. Ikomey George Mondinde., et al. "Evidence of Co and triple infections of Hepatitis B and C amongst HIV infected pregnant women in Buea, Cameroon". (2016).

11. Landrum Michael L., et al. "Hepatitis B vaccination and risk of hepatitis B infection in HIV-infected individuals". AIDS 24.4 (2010): 545.

12. Chiabi Andreas., et al. "Vaccination of infants aged 0 to 11 months at the Yaounde Gynaeco-obstetric and pediatric hospital in Cameroon: how complete and how timely?". BMC Pediatrics 17.1 (2017): 1-7.

13. Xiao Jianpeng., et al. "Impact of hepatitis B vaccination among children in Guangdong Province, China". International Journal of Infectious Diseases 16.9 (2012): e692-e696.

14. Chiaramonte M., et al. "Hepatitis B virus infection in Cameroon: a seroepidemiological survey in city school children". Journal of Medical Virology 33.2 (1991): 95-99.

15. Chasela Charles S., et al. "Hepatitis B virus infection among HIV-infected pregnant women in Malawi and transmission to infants". Journal of Hepatology 60.3 (2014): 508-514.

16. Avdicova Mária., et al. "Lasting immune memory against hepatitis B following challenge 10-11 years after primary vaccination with either three doses of hexavalent DTPa-HBV-IPV/Hib or monovalent hepatitis B vaccine at 3, 5 and 11-12 months of age". Vaccine 33.23 (2015): 2727-2733.

17. Chen Taoyang., et al. "Long-term efficacy of neonatal hepatitis $B$ vaccination against chronic hepatitis $B$ virus infection and

Citation: Lem Edith Abongwa., et al. "Hepatitis B Sero-Conversion Rates among HIV Positive Children Immunized with Pentavalent Vaccine in Bamenda Health District, Cameroon". Acta Scientific Gastrointestinal Disorders 4.8 (2021): 05-10. 
chronic liver disease: a cross-sectional study based on Qidong Hepatitis B Intervention Study". Zhonghua Liu Xing Bing Xue Za Zhi= Zhonghua Liuxingbingxue Zazhi 37.1 (2016): 64-67.

18. Bagheri-Jamebozorgi Masoomeh., et al. "The persistence of anti-HBs antibody and anamnestic response 20 years after primary vaccination with recombinant hepatitis $B$ vaccine at infancy". Human Vaccines and Immunotherapeutics 10.12 (2014): 3731-3736.

19. Chaouch H., et al. "Impact and long-term protection of hepatitis B vaccination: 17 years after universal hepatitis B vaccination in Tunisia". Epidemiology and Infection 144.16 (2016): 3365-3375.

20. Mendy Maimuna., et al. "Observational study of vaccine efficacy 24 years after the start of hepatitis B vaccination in two Gambian villages: no need for a booster dose". PloS one 8.3 (2013): e58029.

21. https://www.who.int/immunization/diseases/hepatitisB/ en/

22. Middleman Amy B., et al. "Duration of protection after infant hepatitis B vaccination series". Pediatrics 133.6 (2014): e1500-e1507.

Volume 4 Issue 8 August 2021

(C) All rights are reserved by Lem Edith Abongwa., et al. 